\title{
Pacific
}

Journal of

Mathematics

\section{ON THE SYMMETRIC SQUARE. UNIT ELEMENTS}

YUVAL ZVI FLICKER 


\title{
ON THE SYMMETRIC SQUARE. UNIT ELEMENTS
}

\author{
Yuval Z. Flicker
}

\begin{abstract}
A twisted analogue of Kazhdan's decomposition of compact elements into a commuting product of topologically unipotent and absolutely semi-simple elements, is developed and used to give a direct and elementary proof of the Langlands' fundamental lemma for the symmetric square lifting from $S L(2)$ to $P G L(3)$ and the unit element of the Hecke algebra. Thus we give a simple proof that the stable twisted orbital integral of the unit element of the Hecke algebra of $P G L(3)$ is suitably related to the stable orbital integral of the unit element of the Hecke algebra of $S L(2)$, while the unstable twisted orbital integral of the unit element on $P G L(3)$ is matched with the orbital integral of the unit element on $P G L(2)$. An Appendix examines the implications of Waldspurger's fundamental lemma in the case of endolifting to the theory of endolifting and that of the metaplectic correspondence for $G L(n)$.
\end{abstract}

Let $F$ be a p-adic field $(p \neq 2)$, and $\bar{F}$ a separable closure of $F$. Put $\mathbf{H}=\mathbf{H}_{0}=S L(2), \mathbf{G}=G L(3) / \mathbf{Z}$, where $\mathbf{Z}$ is the center of $G L(3), J=$

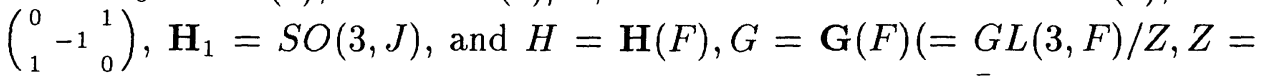
$\mathbf{Z}(F)), H_{1}=\mathbf{H}_{1}(F)$. Put $\sigma(g)=J^{t} \cdot g^{-1} \cdot J$ for $g \in G L(3, \bar{F})$. The elements $\delta, \delta^{\prime}$ of $G$ are called (stably) $\sigma$-conjugate if there is $x$ in $G(\operatorname{resp} \mathbf{G}(\bar{F})$ ) with $\delta^{\prime}=x \delta \sigma\left(x^{-1}\right)$, or $\delta^{\prime} \sigma=\operatorname{Int}(x)(\delta \sigma)$, in the semi-direct product $G \rtimes\langle\sigma\rangle$. The elements $\gamma, \gamma^{\prime}$ of $H$ are called (stably) conjugate if $\gamma^{\prime}=\operatorname{Int}(x) \gamma$ for some $x$ in $H$ (resp. $\mathbf{H}(\bar{F})$ ); similar definitions apply to $H_{1}$. A norm map $N$, from the set of stable $\sigma$-conjugacy classes in $G$, to the set of stable conjugacy classes in $H$, as well as such a map $N_{1}$ to the set of conjugacy classes in $H_{1}$, is defined in [F1]. For any $\delta \in G,(\delta \sigma)^{2}=\delta \sigma(\delta) \in S L(3, F)$ has an eigenvalue 1. If $\delta \sigma(\delta)$ is semi-simple, with eigenvalues $\mu, 1, \mu^{-1}$, then $N \delta$ is the stable class in $H$ with eigenvalues $\mu, \mu^{-1}$.

If $\mu \neq-1$ then $N_{1} \delta$ is the class in $H_{1}$ with eigenvalues $\mu, 1, \mu^{-1}$. Denote by $Z_{G}(\delta \sigma)$ the group of $x$ in $G$ with $\delta \sigma=\operatorname{Int}(x)(\delta \sigma)$, by $Z_{H}(\gamma)$ the centralizer of $\gamma$ in $H$, and by $Z_{H_{1}}\left(\gamma_{1}\right)$ the centralizer of $\gamma_{1}$ in $H_{1}$. For $f \in C_{c}^{\infty}(G)$, $f_{0} \in C_{c}^{\infty}(H), f_{1} \in C_{c}^{\infty}\left(H_{1}\right)$, define the orbital integrals

$$
\Phi_{f}(\delta \sigma)=\int_{G / Z_{G}(\delta \sigma)} f(\operatorname{Int}(x)(\delta \sigma)),
$$




$$
\Phi_{f_{i}}\left(\gamma_{i}\right)=\int_{H_{i} / Z_{H_{i}}\left(\gamma_{i}\right)} f_{i}\left(\operatorname{Int}(x)\left(\gamma_{i}\right)\right) \quad(i=0,1)
$$

where $f(g \sigma)=f(g)$. These depend on choices of Haar measures. The measures on the centralizers are compatible with the isomorphisms $Z_{G}(\delta \sigma) \simeq$ $Z_{H}(N \delta) \simeq Z_{H_{1}}\left(N_{1} \delta\right)$ when $\mu \neq \pm 1$ (in this case $\delta \sigma, \gamma, \gamma_{1}$ are called regular).

Denote by $\left\{\delta^{\prime}\right\}$ a set of representatives for the $\sigma$-conjugacy classes within the stable $\sigma$-conjugacy class of $\delta \in G$; it consists of one or two elements. Define the stable $\sigma$-orbital integral of $f$ at $\delta$ with $\mu \neq \pm 1$ by

$$
\Phi_{f}^{s t}(\delta \sigma)=\sum_{\left\{\delta^{\prime}\right\}} \Phi_{f}\left(\delta^{\prime} \sigma\right)
$$

Similarly put $\Phi_{f_{0}}^{s t}(\gamma)=\sum_{\left\{\gamma^{\prime}\right\}} \Phi_{f_{0}}\left(\gamma^{\prime}\right)$. Define $\Delta(\delta \sigma)=\left|(1+\mu)\left(1+\mu^{-1}\right)\right|^{1 / 2}$. Put $\kappa\left(\delta^{\prime}\right)=1$ if $S O\left(\frac{1}{2}\left[\left(\delta^{\prime} J\right)+{ }^{t}\left(\delta^{\prime} J\right)\right]\right)$ is split, and $\kappa\left(\delta^{\prime}\right)=-1$ otherwise. Define $\Phi_{f}^{\text {us }}(\delta \sigma)$ to be $\Phi_{f}(\delta \sigma)$ if $\mu \in F^{\times}$, and to be $\sum_{\left\{\delta^{\prime}\right\}} \kappa\left(\delta^{\prime}\right) \Phi_{f}\left(\delta^{\prime} \sigma\right)$ if $\mu \notin F^{\times}$. Let $R$ be the ring of integers of $F$. Put $K=\mathbf{G}(R), K_{0}=\mathbf{H}(R), K_{1}=\mathbf{H}_{1}(R)$.

Denote by $f^{0}$ the function on $G$ which is supported on $K$ and whose value there is $1 / \operatorname{vol}(K)=|K|^{-1}$. Denote by $f_{i}^{0}$ the quotient of the characteristic function $\operatorname{ch}_{K_{i}}$ of $K_{i}$ in $H_{i}$ by $\operatorname{vol}\left(K_{i}\right)=\left|K_{i}\right|, i=0,1$. Recall: $p \neq 2$.

Theorem. For $\mu \neq \pm 1$ we have $\Phi_{f^{0}}^{s t}(\delta \sigma)=\Phi_{f_{0}^{0}}^{s t}(N \delta)$. Moreover,

$$
\Delta(\delta \sigma) \Phi_{f^{0}}^{\mathrm{us}}(\delta \sigma)=\Phi_{f_{1}^{\mathrm{o}}}\left(N_{1} \delta\right) \text {. }
$$

This is Langlands' fundamental lemma for the symmetric square lifting from $S L(2)$ to $P G L(3)$ and the unit element of the Hecke algebra. A proof of the first assertion - due to Langlands - is recorded in [F1], $§ 4$, but it is conceptually difficult. In particular, it is based on counting vertices on the Bruhat-Tits buildings associated with $P G L(3)$. The current simpler proof is based on a twisted analogue of Kazhdan's decomposition [K], p. 226, of a compact element into a commuting product of its absolutely semi-simple and its topologically unipotent parts, on an explicit and elementary computation of orbital integrals of the unit element in the Hecke algebra of $G L(2)$, and on the preliminary analysis of stable twisted conjugacy classes from [F1], $\S 1$. For an extension of the Theorem to general spherical functions see [F2], and for representation theoretic applications see [F2] and [F3].

The first draft of the present paper proved the Theorem on using - in addition to the twisted Kazhdan's decomposition and [F1], $§ 1$ - Waldspurger's recent deep coherence result for the germ expansion of the orbital integral of the unit element in the Hecke algebra - see [W1] - on the topologically unipotent set. It states that the local germ expansion of the orbital integral 
of the unit element of the Hecke algebra holds globally. This technique is useful once the germ expansion of the orbital integral is known (in the case of the symmetric square it is given in [F1], §3). In general, the germ expansion might be no easier to obtain than the fundamental lemma itself. The present version is simpler. It needs neither [W1] nor the germ expansion, and it is completely computational and elementary. Of course, no computations on the buildings are needed. We argue that the (twisted) Kazhdan decomposition of Proposition 2 already reduces all computations to $G L(2)$, and we carry out explicitly these computations (in particular explicitly reproving Waldspurger's result in our case). This makes the proof of the fundamental lemma for the symmetric square lifting entirely elementary. Our original motivation to reconsider the proof of [F1], §4, came from reading [W1]. Our final, elementary and purely computational proof, extends to prove the fundamental lemma from $G S p(2)$ to $G L(4)$ twisted by an outer automorphism similar to the one considered here, see [F6].

I wish to use this opportunity to express my deep gratitude to Professors R.P. Langlands for notes on the local theory, which were useful for [F1], and $\mathrm{H}$. Jacquet for notes suggesting to truncate the twisted trace formula by all parabolics. I later realized that the correct truncation should be done using invariant parabolics only. My indebtedness to the work of $\mathrm{D}$. Kazhdan and J.-L. Waldspurger is apparent. I wish to thank R. Weissauer for constructive criticism, DAAD for support during the summer of 1994, and the Humboldt Foundation for support during the summers of 1995 and 1996.

We need a twisted analogue of the following definitions and results of $[\mathbf{K}]$, p. 226.

Put $\mathbb{F}_{q}=R / \pi R$, where $\pi$ generates the maximal ideal in the local ring $R$.

Definition $([\mathbf{K}])$. An element $k \in G=G L(n, F)$ is called absolutely semisimple if $k^{a}=1$ for some positive integer $a$ which is prime to $p$ (= residual characteristic of $F$ ). A $k \in G$ is called topologically unipotent if $k^{q^{N}} \rightarrow 1$ as $N \rightarrow \infty$.

Proposition $1([\mathrm{~K}])$. Any element $k \in K=G L(n, R)$ has a unique decomposition $k=s u=u s$, where $s$ is absolutely semi-simple, $u$ is topologically unipotent, and $s, u$ lie in $K$. For any $k \in K$ and $x \in G$, if $\operatorname{Int}(x) k\left(=x k x^{-1}\right)$ is in $K$, then $x$ lies in $K Z_{G}(s)$; here $Z_{G}(s)$ is the centralizer of $s$ in $G$.

Let $\sigma$ be an automorphism of $G$ of order $\ell,(\ell, p)=1$, whose restriction to $K$ is an automorphism of $K$ of order $\ell$. Denote by $\langle K, \sigma\rangle$ the group generated by $K$ and $\sigma$ in the semi-direct product $G \rtimes\langle\sigma\rangle$.

Definition. The element $k \sigma$ of $G \sigma \subset G \rtimes\langle\sigma\rangle$ is called absolutely semi-simple 
if $(k \sigma)^{a}=1$ for some positive integer $a$ indivisible by $p$.

Proposition 2. Any $k \sigma \in K \sigma$ has a unique decomposition $k \sigma=s \sigma \cdot u=$ $u \cdot s \sigma$ with absolutely semi-simple s $\sigma$ and topologically unipotent $u$. Both $s$ and $u$ lie in $K$.

Definition. This $s \sigma$ is called the absolutely semi-simple part of $k \sigma$ and $u$ is the topologically unipotent part of $k \sigma$.

Proof. For the uniqueness, if $s_{1} \sigma \cdot u_{1}=s_{2} \sigma \cdot u_{2}$ then $u_{1}^{a}=u_{2}^{a}$ for $a=$ $a_{1} a_{2}$. Since $(a, q)=1$, there are integers $\alpha_{N}, \beta_{N}$ with $\alpha_{N} a+\beta_{N} q^{N}=1$. Then $u_{2} u_{1}^{-1}=u_{2}^{\alpha_{N} a+\beta_{N} q^{N}} u_{1}^{-\alpha_{N} a-\beta_{N} q^{N}}=u_{2}^{\beta_{N} q^{N}} u_{1}^{-\beta_{N} q^{N}} \rightarrow 1$ as $N \rightarrow \infty$. For the existence, recall that the prime-to- $p$ part of the number of elements in $G L\left(n, \mathbb{F}_{q}\right)$ is $c=\prod_{i=1}^{n}\left(q^{i}-1\right)$. Let $\left\{(k \sigma)^{q^{m_{i}}}\right\}$ be a convergent subsequence in the sequence $\left\{(k \sigma)^{q^{m}} ; q^{m} \equiv 1(\bmod c \ell)\right\}$ in $\langle K, \sigma\rangle$. Denote the limit by $s \sigma, s \in K$. Then $(s \sigma)^{c \ell}=1$. Define $u=k \sigma(s \sigma)^{-1}$. Then $u^{q^{m_{\imath}}} \rightarrow 1$ as $m_{i} \rightarrow \infty$, and $u^{q^{N}} \rightarrow 1$ as $N \rightarrow \infty$.

Corollary. $Z_{G}(s \sigma \cdot u)$ is contained in $Z_{G}(s \sigma)$.

Proposition 3. Given $k \in K, k \sigma=s \sigma \cdot u$, put $\tilde{\sigma}(h)=s \sigma(h) s^{-1}$. This is an automorphism of order $\ell$ on $Z_{K}\left((s \sigma)^{\ell}\right)$. Suppose that the first cohomology set $H^{1}\left(\langle\tilde{\sigma}\rangle, Z_{K}\left((s \sigma)^{\ell}\right)\right)$, of the group $\langle\tilde{\sigma}\rangle$ generated by $\tilde{\sigma}$, with coefficients in the centralizer $Z_{K}\left((s \sigma)^{\ell}\right)$ of $(s \sigma)^{\ell}$ in $K$, injects in $H^{1}\left(\langle\tilde{\sigma}\rangle, Z_{G}\left((s \sigma)^{\ell}\right)\right)$. Then, any $x \in G$ such that $k^{\prime} \sigma=\operatorname{Int}(x)(k \sigma)$ is in $K \sigma$, must lie in $K Z_{G}(s \sigma)$.

Proof. Put $k^{\prime} \sigma=s^{\prime} \sigma \cdot u^{\prime}$. Then $s^{\prime} \sigma=\lim \left(k^{\prime} \sigma\right)^{q^{m_{2}}}=\operatorname{Int}(x) \lim (k \sigma)^{q^{m_{2}}}=$ $\operatorname{Int}(x)(s \sigma)$. Hence $\left(s^{\prime} \sigma\right)^{\ell}=\operatorname{Int}(x)(s \sigma)^{\ell}$, and by Proposition 1 there is $y \in K$ with $(s \sigma)^{\ell}=\operatorname{Int}(y)\left(s^{\prime} \sigma\right)^{\ell}=(t \sigma)^{\ell}$, where $t=y s^{\prime} \sigma\left(y^{-1}\right)$. Replacing $x$ by $y x$ and $k^{\prime}$ by $y k^{\prime} \sigma\left(y^{-1}\right)$, we may assume that $y=1$. Put $a(1)=1$, and for $0<r<\ell, a\left(\sigma^{r}\right)=s^{\prime} \sigma\left(s^{\prime}\right) \cdots \sigma^{r-1}\left(s^{\prime}\right) \sigma^{r-1}(s)^{-1} \cdots \sigma(s)^{-1} s^{-1}$. Then $a\left(\sigma^{r}\right) \in Z_{K}\left((s \sigma)^{\ell}\right)$, and $a\left(\sigma^{u}\right) \tilde{\sigma}^{u}\left(a\left(\sigma^{r}\right)\right)=a\left(\sigma^{u+r}\right)(0 \leq u, r<\ell)$. Hence $a=$ $\left\{\sigma^{r} \mapsto a\left(\sigma^{r}\right)\right\} \in H^{1}\left(\langle\tilde{\sigma}\rangle, Z_{K}\left((s \sigma)^{\ell}\right)\right)$. Of course, $s^{\prime}=x s \sigma\left(x^{-1}\right)=x \tilde{\sigma}\left(x^{-1}\right) s$ implies that $a(\sigma)=s^{\prime} s^{-1}=x \tilde{\sigma}\left(x^{-1}\right)$, hence $a$ is trivial in $H^{1}\left(\langle\tilde{\sigma}\rangle, Z_{G}\left((s \sigma)^{\ell}\right)\right)$. The injectivity assumption then implies that $s^{\prime} s^{-1}=a(\sigma)$ is $b \tilde{\sigma}\left(b^{-1}\right)=$ $b s \sigma\left(b^{-1}\right) s^{-1}$, and $s^{\prime}=b s \sigma\left(b^{-1}\right)$, with $b \in Z_{K}\left((s \sigma)^{\ell}\right)$. It follows that $\operatorname{Int}(b)(s \sigma)=s^{\prime} \sigma=\operatorname{Int}(x)(s \sigma)$. Hence $b^{-1} x \in Z_{G}(s \sigma)$, and $x \in b Z_{G}(s \sigma) \subset$ $K Z_{G}(s \sigma)$, as required.

Remark. Let us verify the injectivity assumption of Proposition 3 in the case considered in the Theorem. We use the notations and analysis of [F1], (1.4), which implies that the semi-simple element $s \sigma(s)$ in $K$ is the 
identity, or has eigenvalues $-1,1,-1$, or $\lambda, 1, \lambda^{-1}, \lambda^{2} \neq 1$. In the first case $Z_{K}(s \sigma s)=K$, and $I=k \tilde{\sigma} k$ implies $k s J={ }^{t}(k s J)$. This represents a quadratic form in 3 variables over $R$ (= ring of integers in $F$ ), and these are parametrized by their discriminant, in $R^{\times} / R^{\times 2}$. If the form splits over $F$, thus the discriminant lies in $F^{\times 2}$, and in $R^{\times}$, then it lies in $R^{\times 2}$, and the form splits already over $R$. The injectivity follows.

In the second case, replacing $s$ by a $\sigma$-conjugate (see [F1], (1.4.3)), we may assume that $s \sigma(s)=\operatorname{diag}(-1,1,-1)$, and $s=\operatorname{diag}(-1,1,-1)$. Then an element of $Z_{G}(s \sigma(s))$ has the form $a_{1}$ (notations of [F1], (1.4): $a$ in $G L(2, F)$, entries of $a_{1}$ indexed by $(i, j), i+j=$ odd, are 0$)$, and $\tilde{\sigma} a_{1}=\left((\operatorname{det} a)^{-1} a\right)_{1}$. So $1=a_{1} \tilde{\sigma} a_{1}$ means $a^{2}=\operatorname{det} a$, and $a$ is a scalar, in $R^{\times}$. Taking any $h \in G L(2, R)$ with $\operatorname{det} h=a$, we get $h_{1} \tilde{\sigma}\left(h_{1}^{-1}\right)=a_{1}$.

In the third case, $H^{1}\left(\langle\tilde{\sigma}\rangle, Z_{K}\left((s \sigma)^{\ell}\right)\right)$ is trivial (as in the second case) if $\lambda \in R^{\times}$, so let us consider the case where $F(\lambda)$ is a quadratic extension of $F$. As in $[\mathbf{F 1}],(1.5)$, we may assume that $T=Z_{G}(s \sigma(s))$ consists of $b_{1}$, $b \in G L(2, F)$, and $s=(a e)_{1}$. Since $s \sigma(s)=\left(-(\operatorname{det} a)^{-1} a^{2}\right)_{1}, a_{1}$ lies in $T$, and

$$
\tilde{\sigma}(t)=s J^{t} b_{1}^{-1} J s^{-1}=\left(a e w^{t} b^{-1} w e a^{-1}\right)_{1}=\left((\operatorname{det} b)^{-1} a b a^{-1}\right)_{1}=\left((\operatorname{det} b)^{-1} b\right)_{1} .
$$

Hence $1=t \tilde{\sigma}(t)$ means that $b$ is a scalar, in $R^{\times}$. The image in $H^{1}(\langle\tilde{\sigma}\rangle$, $\left.Z_{G}\left((s \sigma)^{\ell}\right)\right)$ is trivial when $b_{1}=c_{1} \tilde{\sigma}\left(c_{1}^{-1}\right)=(\operatorname{det} c)_{1}$, where $c_{1} \in T$, hence $b=\operatorname{det} c$ lies in the norm subgroup $N_{F(\lambda) / F} F(\lambda)^{\times}$, and in $R^{\times}$, hence in $N_{F(\lambda) / F} R(\lambda)^{\times}$, where $R(\lambda)$ denotes the ring of integers of $F(\lambda)$. We conclude that $c$ can be taken in $G L(2, R)$, and $c_{1}$ in $Z_{K}(s \sigma(s))$, as required.

I wish to express my gratitude to R. Weissauer for his help with Proposition 3 .

Proposition 4. If the elements $k \sigma=s \sigma \cdot u$ and $k^{\prime} \sigma=s^{\prime} \sigma \cdot u^{\prime}$ of $K \sigma$ are stably conjugate, then s $\sigma$ and $s^{\prime} \sigma$ are stably conjugate. If $s=s^{\prime}$, then $u, u^{\prime}$ are stably conjugate in $Z_{G}(s \sigma)$.

Proof. Suppose that $k^{\prime} \sigma=\operatorname{Int}(\bar{x})(k \sigma)$ for some $\bar{x} \in \bar{G}=G L(n, \bar{F})$, where $\bar{F}$ is a finite galois extension of $F$ (in the course of this proof). We have the $K$-decomposition $s^{\prime} \sigma \cdot u^{\prime}=\operatorname{Int}(\bar{x})(s \sigma) \cdot \operatorname{Int}(\bar{x}) u$ in $\bar{G}$. The uniqueness of the $K$-decomposition in $\bar{G}$ implies that $s^{\prime} \sigma=\operatorname{Int}(\bar{x})(s \sigma)$, namely $s \sigma, s^{\prime} \sigma$ are stably conjugate. If $s \sigma, s^{\prime} \sigma$ are conjugate, we may assume that $s^{\prime} \sigma=s \sigma$, then $s \sigma \cdot u^{\prime}=\operatorname{Int}(\bar{x})(s \sigma) \cdot \operatorname{Int}(\bar{x}) u$ implies that $\bar{x} \in Z_{\bar{G}}(s \sigma)$ and $\operatorname{Int}(\bar{x}) u=u^{\prime}$, as required.

To prove the Theorem, decompose $k \sigma=s \sigma \cdot u$ (in our case $\sigma(x)=$ $\left.J^{t} \cdot x^{-1} \cdot J^{-1}\right)$. Then $k \sigma(k)=s \sigma(s) \cdot u^{2}$. We shall consider three different 
cases, depending on whether $s \sigma(s)$ is the identity $I$, or it is $\operatorname{diag}(-1,1,-1)$, or it is regular (its eigenvalues $\lambda, 1, \lambda^{-1}$ are distinct). In all cases put

$$
\begin{aligned}
\tilde{f}_{s \sigma}^{0}(u) & =\int_{G / Z_{G}(s \sigma)} f^{0}(\operatorname{Int}(x)(s \sigma \cdot u)) \\
& =\int_{K / K \cap Z_{G}(s \sigma)} f^{0}(\operatorname{Int}(x)(s \sigma \cdot u))=\left|K / K \cap Z_{G}(s \sigma)\right| f^{0}(s \sigma \cdot u),
\end{aligned}
$$

where the second equality follows from Proposition 3 . Note that $\tilde{f}_{s \sigma}^{0}(1)=$ $\Phi_{f^{0}}(s \sigma)$. Then

$$
\begin{aligned}
\Phi_{f^{0}}(k \sigma) & =\int_{G / Z_{G}(k \sigma)} f^{0}(\operatorname{Int}(x)(k \sigma)) \\
& =\int_{Z_{G}(s \sigma) / Z_{G}(s \sigma \cdot u)} \tilde{f}_{s \sigma}^{0}(\operatorname{Int}(x) u)=\Phi_{\tilde{f}_{s \sigma}^{0}}(u) .
\end{aligned}
$$

Here $\Phi_{\tilde{f}_{s \sigma}^{0}}(u)$ denotes the orbital integral of the characteristic function $\tilde{f}_{s \sigma}^{0}$ of the compact subgroup $Z_{K}(s \sigma)=K \cap Z_{G}(s \sigma)$ of $Z_{G}(s \sigma)$ (multiplied by $\left.\left|Z_{K}(s \sigma)\right|^{-1}\right)$ at the topologically unipotent element $u$ in $Z_{K}(s \sigma)$.

Let us compute explicitly the orbital integral of the characteristic function $1_{K}$ of the maximal compact subgroup $K=G L(2, R)$ in $G=G L(2, F)$, where - as usual $-F$ is a local field of odd residual characteristic with ring $R$ of integers. Normalize the Haar measure on $G$ to assign $K$ the volume $|K|=1$. Put $\pi$ for a generator of the maximal ideal in $R, q$ for the cardinality of the residue field $R / \pi R,|\cdot|$ for the normalized (by $|\pi|=q^{-1}$ ) absolute value on $F$. Let $E$ be a quadratic extension of $F$; then $E=F(\sqrt{\theta})$ for some $\theta$ with $|\theta|$ equals 1 or $q^{-1}$. The torus $T=\left\{\gamma=\left(\begin{array}{ll}a & b \theta \\ b & a\end{array}\right) \in G\right\}$ in $G$ is isomorphic to $E^{\times}$, it subgroup $R_{T}=T \cap K$ is isomorphic to $R_{E}^{\times}$, the group of units in $E^{\times}$, via $\gamma \mapsto a+b \sqrt{\theta}$.

Proposition 5. For a regular $(b \neq 0) \gamma$ in $R_{T}$, the orbital integral

$$
\int_{G / T} 1_{K}(\operatorname{Int}(x) \gamma) d x
$$

is equal to $-\frac{2 / e}{q-1}+\frac{q-1+2 / e}{q-1}|b|^{-1}$; here $e=e(E / F)$ is the ramification index of $E$ over $F$. Note that $b=(\gamma-\bar{\gamma}) / 2 \sqrt{\theta}, \bar{\gamma}=a-b \sqrt{\theta}$.

Proof. One has the disjoint decomposition $G=\bigcup_{m \geq 0} K\left(\begin{array}{cc}1 & 0 \\ 0 & \pi^{-m}\end{array}\right) T$, and

$$
K \cap\left(\begin{array}{cc}
1 & 0 \\
0 & \pi^{-m}
\end{array}\right) T\left(\begin{array}{cc}
1 & 0 \\
0 & \pi^{m}
\end{array}\right)=\left\{\left(\begin{array}{cc}
a & \pi^{m} b \theta \\
\pi^{-m} b & a
\end{array}\right) \in K\right\} \simeq R_{E}(m)^{\times}
$$


where $R_{E}(m)=\left\{a+b \sqrt{\theta} ;|b| \leq|\pi|^{m},|a| \leq 1\right\}=R+\pi^{m} R_{E}=R+R \pi^{m} \sqrt{\theta}$. For any function $f \in C_{c}^{\infty}(G / T)$ we then have

$$
\int_{G / T} f(g) d g=\sum_{m \geq 0}\left[R_{E}^{\times}: R_{E}(m)^{\times}\right] \int_{K} f\left(k\left(\begin{array}{cc}
1 & 0 \\
0 & \pi^{-m}
\end{array}\right)\right) d k,
$$

and so

$$
\begin{aligned}
\int_{G / T} 1_{K}(\operatorname{Int}(x) \gamma) d x & =\sum_{m \geq 0}\left[R_{E}^{\times}: R_{E}(m)^{\times}\right] 1_{K}\left(\begin{array}{cc}
a & \pi^{m} b \theta \\
b \pi^{-m} & a
\end{array}\right) \\
& =\sum_{0 \leq m \leq B}\left[R_{E}^{\times}: R_{E}(m)^{\times}\right]
\end{aligned}
$$

if $|b|=\left|\pi^{B}\right|$. Recall that $\pi=\pi_{E}^{e}$ and $q_{E}=q^{2 / e}$ for the uniformizer $\pi_{E}$ and residual cardinality $q_{E}$ of $E$. Since $\left[R_{E}(m)^{\times}: 1+\pi^{m} R_{E}\right]=\left[R^{\times}\right.$: $\left.R^{\times} \cap\left(1+\pi^{m} R_{E}\right)\right]=(q-1) q^{m-1}$, and $\left[R_{E}^{\times}: 1+\pi^{m} R_{E}\right]=\left(q_{E}-1\right) q_{E}^{e m-1}$, we have that $\left[R_{E}^{\times}: R_{E}(m)^{\times}\right]$is $q^{m}$ if $e=2$, while if $e=1$ it is 1 when $m=0$ and $(q+1) q^{m-1}$ when $m \geq 1$. The proposition follows on taking the sum over $0 \leq m \leq B$.

Proof of theorem; stable case. We deal separately with the three cases, where the eigenvalues $\lambda, 1, \lambda^{-1}$ of $s \sigma(s)$ ( $s \sigma$ is the absolutely semi-simple part of $\delta \sigma \in K \sigma)$ have: I. $\lambda=1$; II. $\lambda=-1$; III. $\lambda \neq \pm 1$. Of course, if $\Phi_{f^{0}}(\delta \sigma) \neq 0$, then we may assume that $\delta \in K$.

Case I. By [F1], (1.2), there is one stable conjugacy class of $\delta \in G$ with $(\delta \sigma)^{2}=I$, and it consists of two conjugacy classes, represented by $\sigma$ and by $s^{\prime} \sigma\left(s^{\prime} \in G\right)$. The centralizer $Z_{G}(\sigma)$ of $\sigma$ in $G$ is the split form $S O(2,1)=$ $P G L(2, F)$, while that of $s^{\prime} \sigma, Z_{G}\left(s^{\prime} \sigma\right)$, is the anisotropic form $S O(3)=$ $P D^{\times}, D=$ quaternion algebra over $F$.

Proposition 6. The orbit $\operatorname{Int}(G)\left(s^{\prime} \sigma\right)$ does not intersect $K \sigma$.

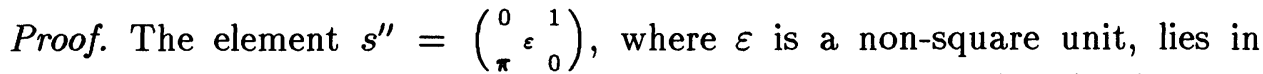
$\operatorname{Int}(G)\left(s^{\prime} \sigma\right)$, since the Witt invariant of $s^{\prime \prime} J=\operatorname{diag}(1,-\varepsilon, \pi)$ is $(\varepsilon, \pi)=-1$. Note that the quadratic form associated to $\operatorname{diag}\left(a_{1}, \ldots, a_{n}\right)$ represents zero precisely when its Witt invariant $\prod_{j \leq i}\left(a_{i}, a_{j}\right)$ is $(-1,-1) ;(\cdot, \cdot)$ denotes the Hilbert symbol. If $s^{\prime}$ lies in $K$, and $s^{\prime} J^{t} s^{\prime} J=1$, namely $s^{\prime} J={ }^{t}\left(s^{\prime} J\right)$, then there is $x \in K$ such that $x s^{\prime} J^{t} x$ is diagonal, $=\operatorname{diag}\left(u_{1}, u_{2}, u_{3}\right)$, in $K$. Its Witt invariant is $\prod_{j \leq i}\left(u_{i}, u_{j}\right)=1=(-1,-1)$. Hence $s^{\prime} J \neq z g s^{\prime \prime} J^{t} g$ for all $g \in G$. 
We conclude that at $\delta \sigma=\sigma \cdot u, u \in K$ topologically unipotent, $u \in$ $S O(2,1)=Z_{G}(\sigma) \simeq P G L(2, F)$, we have $\Phi_{f^{0}}^{s t}(\sigma u)=\Phi_{f^{0}}(\sigma u)=\Phi_{\tilde{f}_{\sigma}^{o}}(u)$. Recall that the eigenvalues of $u \sigma(u)=u^{2}$ are $\mu, 1, \mu^{-1}$. Hence those of $u$ are $\mu^{\prime}, 1, \mu^{\prime-1}$, where $\mu^{\prime}$ is topologically unipotent in $R_{E}^{\times}$with $\mu^{\prime 2}=\mu$. Since $\mu^{\prime} \bar{\mu}^{\prime}=1$, we have $\mu^{\prime}=\nu / \bar{\nu}$ for some topologically unipotent $\nu$ in $R_{E}^{\times}$. Via the isomorphism $S O(2,1) \simeq P G L(2), u$ can be regarded as an element of $P G L(2, R)$ with eigenvalues $\nu, \bar{\nu}$. The integral $\Phi_{\tilde{f}_{\sigma}^{0}}(u)$ is then computed in Proposition 5. It has to be compared with the orbital integral $\Phi_{f_{0}^{0}}^{s t}(v)$ on $S L(2, F)$, where $v$ is an element of $K_{0}=S L(2, R)$ with eigenvalue $\mu, \mu^{-1}$. The stable orbital integral of a function $f_{0}$ on $S L(2, F)$ coincides with its orbital integral over $G L(2, F)$, where $f_{0}$ is extended to a $C_{c}^{\infty}$-function on $G L(2, F)$. This too is computed in Proposition 5. We are reduced then to comparing $\left|(\nu-\bar{\nu})^{2} / \nu \bar{\nu}\right|^{\frac{1}{2}}=|(1-\bar{\nu} / \nu)(\nu / \bar{\nu}-1)|^{\frac{1}{2}}=\left|\left(1-\mu^{\prime}\right)\left(1-\mu^{\prime-1}\right)\right|^{\frac{1}{2}}=$ $\left|(1-\mu)\left(1-\mu^{-1}\right)\right|^{\frac{1}{2}}$ with $\left|\left(\mu-\mu^{-1}\right)^{2}\right|^{\frac{1}{2}}=\left|\left(\mu^{2}-1\right)\left(\mu^{-2}-1\right)\right|^{\frac{1}{2}}$. These are equal since $\nu, \mu^{\prime}, \mu$ are topologically unipotent.

This completes the proof of $\Phi_{f^{0}}^{s t}(\delta \sigma)=\Phi_{f_{0}^{0}}^{s t}(N \delta)$ in Case I.

Case II. Here $\delta \sigma=s \sigma \cdot u, s \sigma(s)$ has eigenvalues $-1,1,-1$. All such $s \in G$ make a single $\sigma$-conjugacy class. Suppose that $\delta^{\prime} \sigma=s^{\prime} \sigma \cdot u^{\prime}=\operatorname{Int}(\bar{x})(\delta \sigma)$ for some $\bar{x} \in \mathbf{G}(\bar{F}), \bar{F}$ finite extension of $F$, with $\delta \sigma, \delta^{\prime} \sigma$ in $K \sigma$. Then $s^{\prime} \sigma=\operatorname{Int}(g)(s \sigma)$ with $g$ in $K$ by Proposition 3. Replacing $\bar{x}$ by $g^{-1} \bar{x}$, we may assume that $s^{\prime}=s=\operatorname{diag}(1,1,-1)$. Then $u, u^{\prime}$ are stably conjugate in $H$. Hence

$$
\Phi_{f_{0}^{0}}^{s t}(\delta \sigma)=\sum_{\left\{u^{\prime}\right\}} \Phi_{f^{0}}\left(s \sigma \cdot u^{\prime}\right)=\sum_{\left\{u^{\prime}\right\}} \Phi_{\tilde{f}_{s \sigma}^{0}}\left(u^{\prime}\right)=\Phi_{\tilde{f}_{s \sigma}^{0}}^{s t}(u)
$$

where $Z_{G}(s \sigma)=H$ and $\tilde{f}_{s \sigma}^{0}=f_{0}^{0}$. We need to compare this with $\Phi_{f_{0}^{0}}^{s t}\left(u^{2}\right)$. Using the explicit computation of Proposition 5 , it suffices to note that for topologically unipotent $\mu$, the value of $\left|\left(\mu-\mu^{-1}\right)^{2}\right|^{1 / 2}$ is equal to that of $\left|\left(\mu^{2}-\mu^{-2}\right)^{2}\right|^{1 / 2}$, since $\left|\left(\mu+\mu^{-1}\right)^{2}\right|=1$. This completes the proof of $\Phi_{f^{0}}^{s t}(\delta \sigma)=\Phi_{f^{0}}^{s t}(N \delta)$ in Case II.

Case III. Here $\delta \sigma=s \sigma \cdot u$, and $s \sigma(s)$ has distinct eigenvalues $\lambda, 1, \lambda^{-1}$. If $\delta \sigma, \delta^{\prime} \sigma$ in $K \sigma$ are stably conjugate but not conjugate, then so are their absolutely semi-simple parts $s \sigma, s^{\prime} \sigma$. Indeed, if $s \sigma, s^{\prime} \sigma$ are conjugate (in $G$ ), then they are so in $K$ by Proposition 3, hence we may assume that $s=s^{\prime}$. If $\delta^{\prime} \sigma=\operatorname{Int}(\bar{x}) \delta \sigma$ then $\bar{x} \in Z_{G}(s \sigma)$, and $u, u^{\prime} \in Z_{G}(s \sigma)$. As $Z_{G}(s \sigma)$ is a torus, $u^{\prime}=u$.

Since $\lambda, \lambda^{-1}$ are absolutely semi-simple and distinct, neither $\lambda$ nor $-\lambda$ are topologically unipotent (as this would imply $\lambda= \pm 1$, and these are cases I, II). It follows that $F(\lambda)$ is not ramified over $F$. Indeed, if it is, $\lambda=a+b \sqrt{\theta}$, 
where $|\theta|=|\pi|,|a|=1,|b| \leq 1$, and $1=\lambda \bar{\lambda}=a^{2}-b^{2} \theta=a^{2}\left(1-\theta(b / a)^{2}\right)$. But $\left(1-\theta(b / a)^{2}\right)^{q^{N}} \rightarrow 1$ as $N \rightarrow \infty$. Hence $a^{2 q^{N}} \rightarrow 1$, and $\pm a=1+\pi c,|c| \leq 1$, for some choice of a sign. Then $\pm a$, and consequently $\pm \lambda$, is topologically unipotent. For the same reason, if $\lambda=a+b \sqrt{\theta},|\theta|=1, \theta \in F-F^{2}$, and $F(\lambda) / F$ is unramified, then $|b|=1$ and $|a| \leq 1$. A set of representatives for the set of $\sigma$-conjugacy classes within the stable $\sigma$-conjugacy class of $\delta$ is given (see [F1], (1.5)) by $\delta_{y}=(y h e)_{1}, e=\left(\begin{array}{cc}-1 & 0 \\ 0 & 1\end{array}\right), h \in G L(2, R)$ (if $g=\left(\begin{array}{ll}a & b \\ c & d\end{array}\right)$ then $\left.g_{1}=\left(\begin{array}{lll}a & 0 & b \\ 0 & 1 & 0 \\ c & 0 & d\end{array}\right)\right)$, as $y$ ranges over a set of representatives of $F^{\times} / N E^{\times}, E=F(\lambda)$. Note that $\delta \sigma(\delta)=\left(\frac{-1}{\operatorname{det} h} h^{2}\right)_{1}$. Take $y=1$ to represent one class. When $F(\lambda) / F$ is unramified, the second representative $y$ is not a unit, hence $\delta_{y} \notin K$, and the stable orbital integral is the sum of a single integral (same conclusion if $\lambda \in F^{\times}$):

$$
\begin{aligned}
\Phi_{f^{0}}^{s t}(\delta \sigma) & =\Phi_{f^{\circ}}(\delta \sigma)=\left|K / K \cap Z_{G}(s \sigma)\right| f^{0}(s \sigma \cdot u) \\
& =\left|K \cap Z_{G}(s \sigma)\right|^{-1}=\left|Z_{K}(s \sigma)\right|^{-1} .
\end{aligned}
$$

The same reasoning implies in our case $(\lambda \neq \pm 1)$ that $\Phi_{f_{0}^{0}}^{s t}(\gamma)=\Phi_{f_{0}^{0}}(\gamma)$, and $\lambda \in F^{\times}$or $F(\lambda) / F$ is unramified, in which case $\gamma$ can be taken to be represented by $\left(\begin{array}{cc}a & b \theta \\ b & a\end{array}\right),|b|=1 \geq|a|$. A stably conjugate, but not conjugate, element, is of the form $\gamma^{\prime}=\operatorname{Int}\left(\begin{array}{ll}1 & 0 \\ 0 & y\end{array}\right)(\gamma)$, with $y \in F-N E, E=F(\lambda)$. In particular $y$ is not a unit, and the conjugacy class of $\gamma^{\prime}$ does not intersect $K_{H}$ (by Proposition 3, and since the eigenvalues of the absolutely semi-simple part $s_{\gamma}$ of $\gamma$ are distinct). Hence

$$
\begin{aligned}
\Phi_{f_{0}^{0}}^{s t}(\gamma)=\Phi_{f_{0}^{0}}(\gamma) & =\int_{H / Z_{H}(\gamma)} f_{0}^{0}\left(\operatorname{Int}(x)\left(s_{\gamma} u_{\gamma}\right)\right) \\
& =\left|K_{0} / K_{0} \cap Z_{H}\left(s_{\gamma}\right)\right| f_{0}^{0}\left(s_{\gamma} u_{\gamma}\right)=\left|K_{0} \cap Z_{H}\left(s_{\gamma}\right)\right|^{-1} .
\end{aligned}
$$

Since $Z_{G}(s \sigma) \simeq Z_{H}\left(s_{\gamma}\right)$, and the measures are chosen in a compatible way, we conclude that $\Phi_{f^{0}}^{s t}(\delta \sigma)=\Phi_{f_{0}^{0}}^{s t}(N \delta)$ in all cases, proving the stable assertion of the theorem.

Proof of theorem; unstable case. Note that if $\mu, 1, \mu^{-1}$ are the (distinct) eigenvalues of the regular $\delta \sigma(\delta), \delta \in K$, then $\mu$ is a unit in $F(\mu)$, and $(1+\mu)\left(1+\mu^{-1}\right)$, which lies in $F$, is a unit in $F$ in cases I and III $(-\mu$ is not topologically unipotent). But in case II we have $\left|(1+\mu)\left(1+\mu^{-1}\right)\right|<1$.

In Case I, by Proposition 6 (and since $\Delta(\delta \sigma)=1$ ) we have the first equality in

$$
\Delta(\delta \sigma) \Phi_{f^{0}}^{\text {us }}(\delta \sigma)=\Phi_{f^{0}}(\sigma u)=\Phi_{\tilde{f}_{\sigma}^{o}}(u)=\Phi_{f_{1}^{0}}(u) .
$$

Here $\delta \sigma=\sigma \cdot u, u$ being topologically unipotent. The second equality follows from $(4.2)$, and $f_{1}^{0}=\tilde{f}_{\sigma}^{0}$ by $(4.1)$. Note that $f_{1}^{0}$ is the characteristic function 
of $K_{1}=K \cap Z_{G}(\sigma)$ in $H_{1}=Z_{G}(\sigma)=S O(2,1)$, divided by the volume of the maximal compact $K_{1}$ of $H_{1}$. Now $N_{1} \delta=u^{2}$. The eigenvalues of $u$, viewed as an element of $P G L(2, R)$, are $\nu, \bar{\nu}$ (topologically unipotent), those of $u^{2}$ are $\nu^{2}, \bar{\nu}^{2}$, and $\left|\left(\nu^{2}-\bar{\nu}^{2}\right)^{2}\right|=\left|(\nu-\bar{\nu})^{2}\right|$, hence Proposition 5 implies that $\Phi_{f_{1}^{0}}\left(u^{2}\right)=\Phi_{f_{1}^{0}}(u)$. Hence $\Delta(\delta \sigma) \Phi_{f^{0}}^{\text {us }}(\delta \sigma)=\Phi_{f_{1}^{0}}(u)=\Phi_{f_{1}^{0}}\left(u^{2}\right)=$ $\Phi_{f_{1}^{0}}\left(N_{1} \delta\right)$.

In Case III, as noted in the discussion of the stable case, $F(\lambda)$ is $F$ or is unramified over $F$, the unstable integral is a sum of a single term, and since $\Delta(\delta \sigma)=1$, we have

$$
\begin{aligned}
\Delta(\delta \sigma) \Phi_{f^{0}}^{\text {us }}(\delta \sigma) & =\Phi_{f^{0}}(\delta \sigma) \\
& =\left|K \cap Z_{G}(s \sigma)\right|^{-1}=\left|K_{1} / K_{1} \cap Z_{H_{1}}\left(s_{\gamma_{1}}\right)\right| f_{1}^{0}\left(\gamma_{1}\right)
\end{aligned}
$$

where $N_{1} \delta=\gamma_{1}$ is the regular class in $H_{1}$ with eigenvalues $\mu, 1, \mu^{-1}$, and $s_{\gamma_{1}}$ is its absolutely semi-simple part. The tori $Z_{G}(s \sigma)$ and $Z_{H_{1}}\left(s_{\gamma_{1}}\right)$ are isomorphic, and the measures are chosen to be compatible with this isomorphism.

In Case II, $\delta \sigma=s \sigma \cdot u \in K \sigma, s \sigma(s)=\operatorname{diag}(-1,1,-1), s=\operatorname{diag}(-1,1,1)$, and $u \in S L(2, R)=Z_{K}(s \sigma)$ has eigenvalues $\gamma, \gamma^{-1}$. Then $\delta \sigma(\delta)$ has eigenvalues $\mu, 1, \mu^{-1}$, where $\mu=-\gamma^{2}$, as does $N_{1} \delta \in S O(2,1)$. Also $\Delta(\delta \sigma)$ is $\left|\left(1-\nu^{2}\right)\left(1-\nu^{-2}\right)\right|^{1 / 2}=\left|\left(\nu-\nu^{-1}\right)^{2}\right|^{1 / 2}$. If $\mu \in F^{\times}$, as an element of $P G L(2, F)$, $\gamma_{1}$ is represented by $\operatorname{diag}(1, \mu)$, and

$$
\begin{aligned}
\Phi_{f_{1}^{0}}\left(\gamma_{1}\right) & =\int_{F} \operatorname{ch}_{K_{1}}\left(\left(\begin{array}{cc}
1 & -x \\
0 & 1
\end{array}\right)\left(\begin{array}{cc}
1 & 0 \\
0 & \mu
\end{array}\right)\left(\begin{array}{ll}
1 & x \\
0 & 1
\end{array}\right)\right) d x \\
& =\int_{F} \operatorname{ch}_{K_{1}}\left(\left(\begin{array}{cc}
1 & 0 \\
0 & \mu
\end{array}\right)\left(\begin{array}{c}
1 \\
0
\end{array}\left(\begin{array}{c}
1-\mu) x \\
1
\end{array}\right)\right) d x=1,\right.
\end{aligned}
$$

where $f_{1}^{0}=\left|K_{1}\right|^{-1} \operatorname{ch}_{K_{1}}, \operatorname{ch}_{K_{1}}$ is the characteristic function of $K_{1}$ in $H_{1}$. Indeed, $-\mu=\nu^{2}$ is topologically unipotent, hence $1-\mu$ (and $\mu$ ) are units in $R$.

If $\mu \notin F$, it lies in a quadratic extension $F(\sqrt{\theta}), \theta \in F-F^{2}$, and we may assume $|\theta|=1$ in the unramified case, and $|\theta|=|\pi|$ in the ramified case. Since $\nu \bar{\nu}=1$, we have $\nu=a+b \sqrt{\theta}$, with $a, b \in R$. Since $\nu$ is topologically unipotent, we have $a \equiv 1(\bmod \pi)$, and $\left|b^{2} \theta\right|<1$. Then $\mu=$ $-\nu / \bar{\nu}=\nu \sqrt{\theta} / \overline{(\nu \sqrt{\theta})}, \nu \sqrt{\theta}=b \theta+a \sqrt{\theta}$, and $\gamma_{1}$, as an element of $H_{1}=$ $P G L(2, F)$, is represented by $\left(\begin{array}{c}b \theta \\ a b \theta \\ b \theta\end{array}\right)$, with eigenvalues $b \theta \pm a \sqrt{\theta}$. In the ramified case, the determinant $b^{2} \theta^{2}-a^{2} \theta$ does not belong to $R^{\times} F^{\times 2}$, hence 
$\Phi_{f_{1}^{0}}\left(\gamma_{1}\right)=0$. In the unramified case, $\gamma_{1}=s_{1} u_{1}$, where the absolutely semisimple part $s_{1}(\in P G L(2, R))$ has eigenvalues whose quotient is -1 . Hence $\Phi_{f_{1}}\left(\gamma_{1}\right)=\left|K_{1} / Z_{K_{1}}\left(s_{1}\right)\right| f_{1}\left(\gamma_{1}\right)=\left|Z_{K_{1}}\left(s_{1}\right)\right|^{-1}$, by Proposition 1 (the integral ranges over the quotient of $K_{1} Z_{H_{1}}\left(s_{1}\right)$ by $Z_{H_{1}}\left(\gamma_{1}\right)$, and $Z_{H_{1}}\left(s_{1}\right)=Z_{H_{1}}\left(\gamma_{1}\right)$ is a torus in $H_{1}$ ).

Let us compare this with $\Delta(\delta \sigma) \Phi_{f^{0}}^{\text {us }}(\delta \sigma)$. If $\nu \in R^{\times}$, then

$$
\begin{aligned}
\Phi_{f^{0}}^{\text {us }}(\delta \sigma)=\Phi_{f^{\mathrm{o}}}(s \sigma \cdot u) & =\int_{G / Z_{G}(s \sigma \cdot u)} f^{0}(\operatorname{Int}(x)(s \sigma \cdot u)) \\
& =\int_{H / Z_{H}(u)} f_{0}^{0}(\operatorname{Int}(x) u) .
\end{aligned}
$$

Here $H=Z_{G}(s \sigma)$, and we used Proposition 3 in the last equality, noting that $f^{0}(1)=|K|^{-1}$ and $f_{0}^{0}(1)=\left|K_{0}\right|^{-1}$. We may represent $u$ by $\operatorname{diag}\left(\nu, \nu^{-1}\right)$, to get

$$
\begin{aligned}
\int_{F} \operatorname{ch}_{K_{0}}\left(\left(\begin{array}{cc}
1 & -x \\
0 & 1
\end{array}\right)\left(\begin{array}{cc}
\nu & 0 \\
0 & \nu^{-1}
\end{array}\right)\left(\begin{array}{ll}
1 & x \\
0 & 1
\end{array}\right)\right) d x & =\int_{F} \operatorname{ch}_{K_{0}}\left(\left(\begin{array}{cc}
\nu & 0 \\
0 & \nu^{-1}
\end{array}\right)\left(\begin{array}{cc}
1 & \left(1-\nu^{-2}\right) x \\
0 & 1
\end{array}\right)\right) d x \\
& =\Delta(\delta \sigma)^{-1}
\end{aligned}
$$

since $\left|1-\nu^{-2}\right|=\left|\nu-\nu^{-1}\right|=\Delta(\delta \sigma)$, and $\nu$ is a unit.

If $\nu \notin F^{\times}$, then the stable conjugacy class of $u$ in $H$ contains a second conjugacy class $u^{\prime}$, represented by $\operatorname{Int}(g) u$, where $g \in \tilde{H}=G L(2, F)$ has $\operatorname{det} g \in F-N E, E=F(\nu)$; here $N E=\operatorname{Norm}_{E / F} E$. Then

$$
\begin{aligned}
\Phi_{f^{0}}^{\text {us }}(\delta \sigma) & =\Phi_{f^{0}}(s \sigma \cdot u)-\Phi_{f^{0}}\left(s \sigma \cdot u^{\prime}\right) \\
& =\int_{H / Z_{H}(u)} f_{0}^{0}(\operatorname{Int}(x) u) d x-\int_{H / Z_{H}(u)} f_{0}^{0}(\operatorname{Int}(g x) u) d x
\end{aligned}
$$

is zero when $F(\nu)$ is ramified over $F$, since $g$ can be chosen in $K_{0}$, with $\operatorname{det} g$ in $R^{\times}-R^{\times} \cap N E$, in this case. When $F(\nu)$ is unramified over $F$, we have that $N E^{\times}=\pi^{2 \mathbb{Z}} R^{\times} \supset R^{\times}$. Since $H / Z_{H}(u)$ is open in $\tilde{H} / Z_{\tilde{H}}(u)$, the measure on $H / Z_{H}(u)$ defines one on $\tilde{H} / Z_{\tilde{H}}(u)$, and if $\varepsilon$ denotes the character of $F^{\times}$ whose kernel is $N E^{\times}$(this is the unramified character of $F^{\times}$of order exactly two), then

$$
\Phi_{f^{0}}^{\mathrm{us}}(\delta \sigma)=\int_{\tilde{H} / Z_{\tilde{H}}(u)} f_{0}^{0}(\operatorname{Int}(x) u) \varepsilon(\operatorname{det} x) d x .
$$

We may represent the topologically unipotent element $u$ by $\left(\begin{array}{c}a b \theta \\ b\end{array}\right.$ $R^{\times 2}$.

It is important to note that $\delta \sigma=s \sigma \cdot u=u \cdot s \sigma$ with

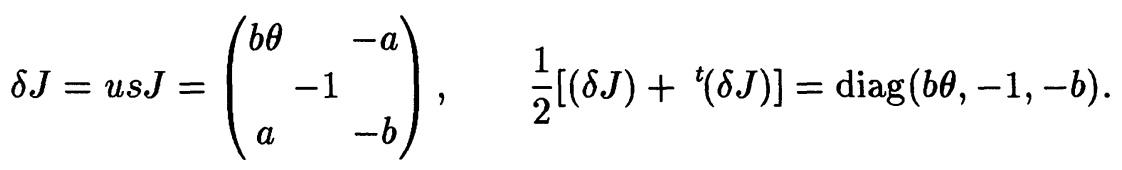


The quadratic form associated to $\operatorname{diag}\left(a_{1}, \ldots, a_{n}\right)$ represents 0 precisely when $\prod_{j \leq i}\left(a_{i}, a_{j}\right)$ is equal to $(-1,-1)$, where $(\cdot, \cdot)$, is the Hilbert symbol. Hence $\kappa(\delta)$ is 1 , and $S O(\operatorname{diag}(b \theta,-1,-b))$ splits, precisely when $(-b, \theta)=1$. In our unramified case this happens precisely when $b \in \pi^{2 \mathbb{Z}} R^{\times}$. Hence $\varepsilon(b)=1$. Note that

$\Delta(\delta \sigma)=\left|\left(1-\nu^{2}\right)\left(1-\nu^{-2}\right)\right|^{1 / 2}=\left|\left(\nu-\nu^{-1}\right)^{2}\right|^{1 / 2}=\left|(\nu-\bar{\nu})^{2}\right|^{1 / 2}=\left|4 b^{2} \theta\right|^{1 / 2}=|b|$.

Now if $\tilde{T}=Z_{\tilde{H}}(u), \tilde{K}=G L(2, R)$, the following is well known.

Lemma 7. The group $\tilde{H}=G L(2, F)$ is equal to $\bigcup_{m \geq 0} \tilde{K}\left(\begin{array}{cc}1 & 0 \\ 0 & x^{-m}\end{array}\right) \tilde{T}$. When $F(u) / F$ is unramified, $\delta_{m}=\left[\tilde{K}\left(\begin{array}{cc}1 & 0 \\ 0 & \pi^{-m}\end{array}\right) \tilde{T}: \tilde{Z} \tilde{K}\right]$ is 1 if $m=0$ and $q^{m}(1+$ $\left.q^{-1}\right)$ if $m>0$. When $F(u) / F$ is ramified, $\delta_{m}=q^{m}$.

Proof. If $\tilde{T}=\left\{\left(\begin{array}{cc}a & b \theta \\ b & a\end{array}\right)\right\}$, put $\tilde{T}_{m}=\left\{\left(\begin{array}{cc}1 & 0 \\ 0 & \pi^{-m}\end{array}\right)\left(\begin{array}{cc}a & b \theta \\ b & a\end{array}\right)\left(\begin{array}{cc}1 & 0 \\ 0 & \pi^{m}\end{array}\right)\right\}=\left\{\left(\begin{array}{cc}a \\ b \pi^{-m} & b \theta \boldsymbol{\pi}^{m} \\ a\end{array}\right)\right\}$. Then $\delta_{m}=\left[\tilde{K} \cdot \tilde{T}_{m}: \tilde{Z} \tilde{K}\right]=\left[\tilde{T}_{m}: \tilde{T}_{m} \cap \tilde{Z} \tilde{K}\right]$ is isomorphic to the quotient of the subgroup $\left\{a+b \sqrt{\theta} ;\left|a^{2}-b^{2} \theta\right|=1\right\}$ of $F(\theta)^{\times}$, by its subgroup which is defined by $|b| \leq|\pi|^{m}$. Representatives for this quotient are given by $a+b \sqrt{\theta}$, $a=1$ and $b$ in $R / \pi^{m} R$ (if $|a|=1$ ), and by $a+b \sqrt{\theta}, b=1$ (if $|a|<1$ ) and $a$ in $\pi R / \pi^{m} R$ if $F(\theta) / F$ is unramified; $|a|=1$ if $F(\theta) / F$ is ramified.

Put $t=\left|K_{0} / Z_{K_{0}}(u)\right|$. Then

$$
\begin{aligned}
& \Delta(\delta \sigma) \Phi_{f^{0}}^{\text {us }}(\delta \sigma)=t \sum_{m=0}^{\infty} \delta_{m} \varepsilon\left(b \pi^{-m}\right)|b| f_{0}^{0}\left(\left(\begin{array}{cc}
a & b \theta \pi^{m} \\
b \pi^{-m} & a
\end{array}\right)\right) \\
& =t \varepsilon(b)|b| f_{0}^{0}\left(\left(\begin{array}{cc}
a & b \theta \\
b & a
\end{array}\right)\right)+t\left(1+q^{-1}\right) \sum_{m=1}^{\infty} \varepsilon\left(b \pi^{-m}\right)\left|b \pi^{-m}\right| f_{0}^{0}\left(\left(\begin{array}{cc}
a & b \theta \pi^{m} \\
b \pi^{-m} & a
\end{array}\right)\right) \\
& =\left[(-1)^{n} q^{-n}+\left(1+q^{-1}\right) \sum_{m=1}^{n}(-1)^{n-m} q^{m-n}\right]\left|Z_{K_{0}}(u)\right|^{-1} \\
& =\left|Z_{K_{0}}(u)\right|^{-1}, \quad|b|=\left|\pi^{n}\right| .
\end{aligned}
$$

Since $Z_{H}(u)$ and $Z_{H_{1}}\left(\gamma_{1}\right)$ are isomorphic tori, and the measures are chosen in a compatible way, the theorem follows in the unstable case II as well, as required.

Remark (1). We sketch the well-known germ expansion of orbital integrals (cf. Shalika [Sh], Vigneras [V]).

For any $g$ in $G$, the centralizer $Z_{G}(g)$ of $g$ in $G$ is unimodular (see, e.g., Springer-Steinberg [SS], III, (3.27b), p. 234). By Bernstein-Zelevinski [BZ], (1.21), it follows that there is a unique (up to a scalar multiple) non zero 
measure (positive distribution) on every $\operatorname{Int}(G)$-orbit $\mathcal{O}$. By Rao $[\mathbf{R}]$ for a general $G$ in characteristic zero, and Bernstein [B], (4.3), p. 70, for $G=$ $G L(n)$ in any characteristic, this extends to a unique (non zero) $\operatorname{Int}(G)$ invariant measure $\Phi_{\mathcal{O}}$ on $G$ whose support is the closure $\overline{\mathcal{O}}$ of $\mathcal{O}$ in $G$ ( $\Phi_{\mathcal{O}}$ is the orbital integral over $\mathcal{O}$; it is a linear form on $C_{c}^{\infty}(G)$ - not only $C_{c}^{\infty}(\mathcal{O})$ - which takes positive values at positive valued functions).

Let $s$ be a semi-simple element in a $p$-adic reductive group $G$. Its centralizer $Z_{G}(s)$ in $G$ is reductive, and also connected when the derived group of $G$ is simply connected ([SS], II, (3.19), p. 201). Lemma 19 of Harish-Chandra $[\mathbf{H C}]$, p. 52 , can be used to reduce the $G$-orbital integrals near $s$ to $Z_{G}(s)$ orbital integrals near the identity. Except in the next Lemma, only the case of $s=$ identity is used below.

Let $X$ be the closed (see, e.g., [SS], III, Theorem 1.8(a), p. 217) set of the elements in $G$ whose semi-simple part is in $\operatorname{Int}(G) s$. There are only finitely many $\operatorname{Int}(G)$-orbits $\mathcal{O}$ in $X$ (see Richardson [Ri], Proposition 5.2, and Serre [S], III, 4.4, Cor. 2). Since $\mathcal{O}$ is open in $\overline{\mathcal{O}}$, and $\operatorname{dim} \mathcal{O}^{\prime}<\operatorname{dim} \mathcal{O}$ for every orbit $\mathcal{O}^{\prime} \subset \overline{\mathcal{O}}, \mathcal{O}^{\prime} \neq \mathcal{O}$ (see Borel [Bo], I.1.8 ("Closed Orbit Lemma"), and Harish-Chandra [HC], Lemma 31, p. 71), there are $f_{\mathcal{O}} \in C_{c}^{\infty}(G)$ with $\Phi_{\mathcal{O}}\left(f_{\mathcal{O}^{\prime}}\right)=\delta_{\mathcal{O}, \mathcal{O}^{\prime}}$ for all orbits $\mathcal{O}, \mathcal{O}^{\prime}$ in $X$. In fact, the $\mathcal{O}$ can be numbered $\mathcal{O}_{i}(1 \leq i \leq k)$, with $\mathcal{O}_{1}=\operatorname{Int}(G) s, \mathcal{O}^{j}=\cup_{i \leq j} \mathcal{O}_{i}$ closed in $G$, and $\mathcal{O}_{j}$ open in $\mathcal{O}^{j}$ for all $j$. The $f_{\mathcal{O}_{j}}$ can then be chosen to be zero on $\mathcal{O}_{i}(i<j)$. We may subtract a multiple of $f_{\mathcal{O}_{\mathfrak{z}}}(i>j)$ to have $\Phi_{\mathcal{O}_{\mathfrak{z}}}\left(f_{\mathcal{O}_{j}}\right)=0$ also for $i>j$.

Lemma. For every $f \in C_{c}^{\infty}(G)$ there exists a (G-invariant) neighborhood $V_{f}$ of the identity in $G$, such that the orbital integral $\Phi(\gamma, f)$ of $f$ is equal to $\sum_{\mathcal{O}} \Phi_{\mathcal{O}}(f) \Phi\left(\gamma, f_{\mathcal{O}}\right)$ for all $\gamma$ in $V_{f}$. The germ $\Gamma_{\mathcal{O}}(\gamma)$ of $\Phi\left(\gamma, f_{\mathcal{O}}\right)$ at the identity in $G$ is independent of the choice of $f_{\mathcal{O}}$.

Proof. The function $f^{\prime}=f-\sum_{\mathcal{O}} \Phi_{\mathcal{O}}(f) f_{\mathcal{O}}$ satisfies $\Phi_{\mathcal{O}}\left(f^{\prime}\right)=0$ for all $\mathcal{O} \subset X$. Denote by $C_{c}^{\infty}(X)^{*}$ the space of distributions on $X$, and by $C_{c}^{\infty}(X)^{* G}$ the subspace of $\operatorname{Int}(G)$-invariant ones. Denote by $C_{c}^{\infty}(X)_{0}$ the span of $\hbar-g \cdot \hbar$ $\left(\hbar \in C_{c}^{\infty}(X), g \in G\right)$, where $g \cdot \hbar(x)=\hbar\left(\operatorname{Int}\left(g^{-1}\right) x\right)$. Then $C_{c}^{\infty}(X)^{* G}=$ $\left(C_{c}^{\infty}(X) / C_{c}^{\infty}(X)_{0}\right)^{*}$. The $\Phi_{\mathcal{O}}$ span $C_{c}^{\infty}(X)^{* G}$. Hence $f^{\prime}$ is annihilated by any element of $\left(C_{c}^{\infty}(X) / C_{c}^{\infty}(X)_{0}\right)^{*}$. Then the restriction $\bar{f}^{\prime}$ of $f^{\prime}$ to the closed subset $X$ (see [BZ], (1.8)) is in $C_{c}^{\infty}(X)_{0}$. Hence there are finitely many $\hbar_{i}$ in $C_{c}^{\infty}(X)$, and $g_{i} \in G$, with $\bar{f}^{\prime}=\sum_{i}\left(\hbar_{i}-g_{i} \cdot \hbar_{i}\right)$. Extend (by [BZ], (1.8)) $\hbar_{i}$ to elements $h_{i}$ of $C_{c}^{\infty}(G)$. Then $f-\sum_{\mathcal{O}} \Phi_{\mathcal{O}}(f) f_{\mathcal{O}}-\sum_{i}\left(h_{i}-g_{i} \cdot h_{i}\right)$ is (compactly) supported in the ( $G$-invariant) open set $G-X$. Hence there is a ( $G$-invariant) neighborhood $V_{f}$ of the identity in $G$ where $f=\sum_{\mathcal{O}} \Phi_{\mathcal{O}}(f) f_{\mathcal{O}}+\sum_{i}\left(h_{i}-g_{i} \cdot h_{i}\right)$, and the lemma follows. 
(2). Waldspurger's homogeneity result which was used in the first draft of this paper, is the following. Let $G$ be any of the groups considered in [W1] (these include all the groups considered here) $\mathfrak{g}$ its Lie algebra, $K$ a standard maximal compact subgroup (i.e. the fixer of each point of a fixed face of minimal dimension in the building of the reductive connected $F$-group $\mathbf{G}$ whose group of $F$-points is $G$ ), and $\mathfrak{k}$ its Lie algebra (which is a sub- $R$ algebra of $\mathfrak{g})$. Denote by $\mathrm{ch}_{K}$ and $\mathrm{ch}_{\mathfrak{k}}$ the characteristic functions of $K$ in $G$ and $\mathfrak{k}$ in $\mathfrak{g}$. Then [W1] defines an isomorphism $e: \mathfrak{g}_{t n} \rightarrow G_{t u}$ from the set $\mathfrak{g}_{t n}=\left\{X \in \mathfrak{g} ; \lim _{N \rightarrow \infty} X^{N}=0\right\}$ of topologically nilpotent elements of $\mathfrak{g}$ to the set $G_{t u}=\left\{u \in G ; \lim _{N \rightarrow \infty} u^{q^{N}}=1\right\}$ of topologically unipotent elements in $G$, named the truncated exponential map. Let $\mathcal{O}_{\text {nil }}$ denote the set of nilpotent orbits in $\mathfrak{g}$. For each $\mathcal{O} \in \mathcal{O}_{\text {nil }}$ fix a $G$-invariant measure on $\mathcal{O}$, and denote by $\Phi_{\mathcal{O}}(f)$ the orbital integral of $f \in C_{c}^{\infty}(\mathfrak{g})$ over $\mathcal{O}$. Fix a maximal $F$-torus $T$, let $\mathfrak{t}$ be its Lie algebra, and denote by $T_{\text {reg }}$ and $\mathfrak{t}_{\text {reg }}$ their regular subsets. For each $\mathcal{O} \in \mathcal{O}_{\text {nil }}$ there exists a unique real positive valued function $\Gamma_{\mathcal{O}}^{T}$ on $\mathfrak{t}_{\text {reg }}$ satisfying the homogeneity relation $\Gamma_{\mathcal{O}}^{T}\left(\mu^{2} H\right)=|\mu|^{-\operatorname{dim} \mathcal{O}} \Gamma_{\mathcal{O}}^{T}(H)$ for all $\mu \in F^{\times}, H \in \mathfrak{t}_{\text {reg }}$, and such that for each $f \in C_{c}^{\infty}(\mathfrak{g})$ one has that the orbital integral $\Phi_{f}(H)=\int_{G / Z_{G}(H)} f(\operatorname{Int}(x) H)$ is equal to $\sum_{\mathcal{O} \in \mathcal{O}_{\text {nil }}} \Gamma_{\mathcal{O}}^{T}(H) \Phi_{\mathcal{O}}(f)$ for each $H$ in a neighborhood of 0 in $\mathfrak{t}_{\text {reg. }}$. Waldspurger's fundamental coherence result - which is no longer used in our proof - is the following (see [W1], Proposition V.3 and V.5).

Proposition ([W1]). For a sufficiently large $p$, for any $H$ in $\mathfrak{t}_{\text {reg }} \cap \mathfrak{g}_{t n}$, we have

$$
\Phi\left(e(H), \operatorname{ch}_{K}\right)=\sum_{\mathcal{O} \in \mathcal{O}_{\text {nil }}} \Gamma_{\mathcal{O}}^{T}(H) \Phi_{\mathcal{O}}\left(\operatorname{ch}_{\mathfrak{k}}\right)
$$

This sum can equivalently be expressed as a sum over the unipotent conjugacy classes in $G$, in which case $\Phi_{\mathcal{O}}\left(\mathrm{ch}_{\mathfrak{t}}\right)$ can be replaced by $\Phi_{\mathcal{O}}\left(\mathrm{ch}_{K}\right)$, and $\Gamma_{\mathcal{O}}^{T}(H)$ can be viewed as a function of $e(H)$ on $G_{t u} \cap T_{\text {reg. }}$.

\section{Appendix. On the transfer sign of endo-lifting.}

The metaplectic correspondence $[\mathbf{F K}]$ relates admissible, and automorphic, representations of $G L(r)$ over a local or global field $F$ containing a primitive $n$th root of 1 , with such representations of a topological $n$-fold metaplectic covering group $\tilde{G}$ of $G L(r)$. The proof in $[\mathbf{F K}]$ is based on a comparison of trace formulae, and a key technical tool is a transfer of orbital integrals of general and spherical test functions, when $F$ is local. The statement of this transfer requires a definition of a map $t: G L(r, F) \rightarrow \tilde{G}$, introduced in Kazhdan-Patterson [KP], Theorem 4.1, p. 200 (and [F5], p. 130, when 
$r=2$ ). This map consists of raising a section to the $n$th power, and multiplying by a sign $u(g)$ when $n$ is even. The function $u: G_{0} \rightarrow\{ \pm 1\}$, where $G_{0}$ is the set of $g \in G L(r, F)$ with $x_{i}+x_{j} \neq 0\left(x_{1}, \ldots, x_{r}\right.$ are the eigenvalues of $g$ ), is introduced in [KP], Theorem 2.1, p. 181, where its main properties are listed. Theorem 5.2 , p. 211 , of $[\mathbf{K P}]$, reduces the transfer of orbital integrals of the unit elements in the Hecke algebras (= characteristic functions of the standard maximal compact subgroups), in this case of the metaplectic correspondence, to the transfer of orbital integrals of the unit elements in the endoscopic lifting from $H=G L\left(r^{\prime}, E\right)$ to $G=G L\left(n^{\prime}, F\right)$, where $E / F$ is a cyclic extension with $r^{\prime}[E: F]=n^{\prime}$. The endoscopic transfer of orbital integrals, for general and spherical (in particular, the unit elements) functions, was proven when $r^{\prime}=1$ by Kazhdan [K]. Section 12 of [FK] rewrote the reduction of $[\mathbf{K P}]$, Theorem 5.2, of the metaplectic transfer for the unit elements. Theorem 19, p. 83 , of $[\mathbf{F K}]$, reduces the metaplectic transfer of orbital integrals of spherical functions to the case of the unit elements, by means of a global technique based on the usage of "regular functions", first introduced in [FK]. Section 13 of [FK] reduces the metaplectic transfer of orbital integrals of general test functions, to the endoscopic transfer of general test functions (done in $[\mathrm{K}]$ when $r^{\prime}=1$ ).

It is noted in [F4], Proposition $\left[C_{0} \Rightarrow C\right]$, p. 281, that the regular functions technique of [FK], Theorem 19, reduces the endoscopic transfer of all spherical functions ("Conjecture C" in [F4], due to [K] when $r^{\prime}=1$ ), to the case of the unit element ("Conjecture $C_{0}$ " in [F4]). It is noted in [F4], Proposition $[\mathrm{C} \Rightarrow \mathrm{B}]$, p. 281, that since the rigidity ("strong multiplicity one") theorem holds for GL, standard trace formula techniques show that this "Conjecture C" for all $\mathrm{F}$ of sufficiently large residual characteristic implies "Conjecture B" of [F4], p. 278 (for all local fields $F$ ), which asserts the existence of an endoscopic lifting of representations of $G L\left(r^{\prime}, E\right)$ to representations of $G L\left(n^{\prime}, F\right)$. Using the trace Paley-Wiener theorem of BernsteinDeligne-Kazhdan [BDK], Proposition $[B \Rightarrow A]$ of [F4], p. 278, shows that the lifting of "Conjecture B" implies the endoscopic transfer of general test functions, stated as "Conjecture A" in [F4], p. 276. As noted above, this implies the metaplectic transfer of orbital integrals, and consequently the metaplectic correspondence.

In a deep and beautiful purely local work, Waldspurger [W2] proved Conjecture $C_{0}$ of [F4] (see Théorème, [W2], p. 852; the restriction of moderate ramification put there can be removed on using the global techniques of [FK], Theorem 19). As noted above, all other assertions concerning the endoscopic and metaplectic correspondences follow from this fundamental result. (Theorem 1, p. 282, of [F4], shows that "Conjecture $C_{0}$ " follows by global techniques from a computation of a twisted character of a specific rep- 
resentation ("Conjecture $B_{0}$ "), suggesting another possible initial approach to the proof of these transfers and liftings.)

The purpose of this Appendix is then to point out these implications of Waldspurger's theorem. Moreover, the sign which appears in the statement of the endoscopic transfer of orbital integrals in [W2] (is correct, suggested by Hales $[\mathbf{H}]$, and) is different from that in $[\mathbf{K}],[\mathbf{K P}],[\mathbf{F K}]$ and $[\mathbf{F 4}]$. Our main purpose is then to correct the choice of sign, starting from [KP], and the formal implications of this change in $[\mathbf{F K}]$ and $[\mathbf{F 4}]$. On the other hand, the sign of [W2] is defined only in the case of an unramified extension $\mathrm{E} / \mathrm{F}$. We shall redefine the sign of [W2] so that it makes sense globally, including the ramified places.

Corollary 2.2 of [KP], p. 187, computes the sign $u(j(x))$, for an elliptic compact element $x=\sigma \tau$ ( $\tau$ : topologically unipotent; $\sigma$ : absolutely semisimple; as introduced in $[K]$, p. 226), when E/F is unramified. The (correct) computation (p. 188, $\ell .11$, to mid-page 189), gives the answer stated on p. $188, \ell$. 10 , which coincides with the $\operatorname{sign} \Delta_{G}^{H, 2}(\gamma)$ of [W2], last line of page 857. But the passage from $[\mathbf{K P}]$, p. $187, \ell$. -3 , to p. $188, \ell .10$, is correct only when $r$ is not divisible by 4 (as indeed stated on p. 188, $\ell .6 / 7$ ). So erase p. $187, \ell .-3$, to p. $188, \ell .9$, and replace "Next we" on $\ell .11$ by "Proof. We". In this context, on $\ell$. 13 , replace " $0 \leq i<r$ " by " $0<i<r$ " on $\ell$. 20, " $2 / 2$ " by " $r / 2$ ", erase "(" on $\ell$. -1 . For the passage from $\ell .6$ to $\ell$. 7 on p. 189 , note that $(-1, \pi)_{F}$ is 1 precisely when -1 is a square in $\mathbb{F}_{q}$, thus $q \equiv 1(\bmod 4)$, hence $(-1, \pi)_{F}=(-1)^{(q-1) / 2}$; when $F$ is replaced by $E$, $q$ is replaced by $q^{r}\left(q^{r / 2}\right.$ for $\left.E_{1}\right)$. To pass from $\ell$. -8 to $\ell$. -7 on p. 188, note that $(\sigma, \pi)_{E}=\left(\sigma \mathrm{Fr}^{r / 2} \sigma, \pi\right)_{E_{1}}=(-1)^{\left(q^{r / 2}-1\right) / 2}\left(\sigma^{2}, \pi\right)_{E_{1}}$, and that $\sigma^{2}$ is not a square in $E_{1}$.

"Assertion $\left(F^{\prime}, F, r^{\prime}\right)$ " of [FK], p. 67 , is wrongly stated (as is " $B C(E / F, \ell)$ " of [KP], p. 211). Its second line, before "we have," should be: "and any $K$-semi-simple $s$ in $K=G L\left(r, R_{F}\right)$ such that $G_{s}=G^{\prime}=G L\left(r^{\prime}, F^{\prime}\right)$, and with $\varepsilon(x)=\left(s^{n}, x\right)^{-1}$ ". Further, " $u(k)^{m-1}$ " on the next line should be " $u(s k)^{m-1}$ ". By virtue of the corrected Corollary 2.2 of $[\mathbf{K P}]$, this coincides with the sign of [W2]. Also, " $f_{F}^{0}$ " on p. $67, \ell .12$, should be " $f$ ", and on $\ell$. -3 , insert " $f^{\prime}=\left[F^{\prime}: F\right]$ " after " $r$ ' $f^{\prime}=r$ ". The proof of the Proposition on p. $68, \ell .1$, that: " "Assertion $\left(F^{\prime \prime}, F^{\prime}, r^{\prime}\right)$ " for all $\left[F^{\prime \prime}: F^{\prime}\right] r^{\prime \prime} \leq r$ implies $(*)$, is valid as it stands. But note that the $k$ on p. $68, \ell .-12$, is $k=s u$; this topologically unipotent $u$ is denoted by $k$ in the statement of "Assertion $\left(F^{\prime \prime}, F^{\prime}, r^{\prime}\right)$ " on p. 67 . Let us explain why " $u(k)=1$ if $n$ is even and $m$ is odd," on p. $68, \ell .13 / 14$ (on $\ell .14$, "[KP']" should be "[KP]"): If $u(k) \neq 1$, by $[\mathbf{K P}]$, Corollary 2.2, $r$ is even, and $\mathrm{Fr}^{r / 2} s=-s$; hence $F(s)=E$. If $m$ is odd, then $\mathrm{Fr}^{r / 2} s^{m}=-s^{m}$, hence $E=F\left(s^{m}\right)=F^{\prime}$, so $r^{\prime}=1$, and $Z_{G}\left(s^{n}\right)=F^{\times}=E^{\times}$, namely $F\left(s^{n}\right)=E$. But $\mathrm{Fr}^{r / 2} s^{2}=s^{2}$ implies that 
$\mathrm{Fr}^{r / 2} s^{n}=s^{n}$, hence $F\left(s^{n}\right) \subset E_{1} \varsubsetneqq E$. This contradiction implies that $u(k)=1$, as asserted. Also on [FK], p. 70, $\ell$. 6, erase ", with determinant in $F^{\times n}$ "; on $\ell$. 18 , replace "non-trivial" by "primitive". Finally, on p. $71, \ell$. 2 , replace " $\varepsilon^{\prime}\left(\tilde{\Delta}(g) / g^{0}\right)^{n(n-1) / 2}$ ", by " $\varepsilon_{1}\left(\bar{\Delta}(g) / \bar{\Delta}\left(g^{0}\right)\right)^{m-1}$, up to a multiple $( \pm 1)$ which depends only on $\varepsilon$ ". Here $\varepsilon_{1}$ is the non-trivial quadratic character of $F_{1}^{\prime \prime x}$ whose kernel is $N_{F^{\prime \prime}} / F_{1}^{\prime \prime} F^{\prime \prime x}$, when $m=\left[F^{\prime \prime}: F^{\prime}\right]$ is even, and $F_{1}^{\prime \prime}$ $\left(F^{\prime} \subset F_{1}^{\prime \prime} \subset F^{\prime \prime}\right)$ is the fixed field of $\sigma^{m / 2}\left(\epsilon\langle\sigma\rangle=\operatorname{Gal}\left(F^{\prime \prime} / F^{\prime}\right)\right)$ in $F^{\prime \prime}$. The object $\bar{\Delta}(g) \in F^{\prime \prime x}$ is defined below. The computation of $u_{\varepsilon}(g)$ of p. $71, \ell$. 2 , follows closely that of [KP], Corollary 2.2 .

We now proceed to define the sign of the transfer factor of the endoscopic lifting, and to indicate the implied changes which need to be made in [F4], Section I. On p. 274, between $\ell$. 12 and 13, insert: "If $k=[E: F]$ is odd, put $\kappa_{+}=1$. If $k$ is even, put $\sigma_{+}=\sigma^{k / 2}, E_{+}=$the field of $x \in E$ fixed by $\sigma_{+}, \kappa_{+}=$the non-trivial character of $E_{+}^{\times} / N_{E / E_{+}} E^{\times}$." On $\ell$. 14, replace

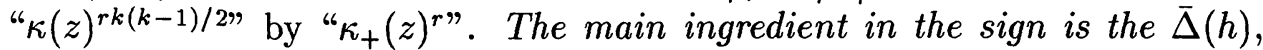
whose definition (on p. $275, \ell .8 / 9$ ), has to be replaced by: " $\Pi\left(h_{\ell}-\sigma_{+} h_{\ell^{\prime}}\right)$, with product ranging over all $\ell, \ell^{\prime}$ with $1 \leq \ell, \ell^{\prime} \leq r$." On $\ell$. 14, the relation should be " $\sigma_{+} \bar{\Delta}(h)=(-1)^{r} \bar{\Delta}(h)$." On $\ell .15$, " $\tilde{\Delta}(h) \neq 0$ ". On $\ell .16$ (twice) and 17 , replace $F$ by $E_{+}$, and note that $\sigma_{+}$generates $\operatorname{Gal}\left(E / E_{+}\right)$. The transfer factor, on $\ell$. 20 , should be: " $\Delta(h)=|\tilde{\Delta}(h)| \kappa_{+}\left(\bar{\Delta}(h) / \bar{\Delta}\left(h_{0}\right)\right)$ ". On $\ell$. 21 , erase: "or divisible by 4 ", and on $\ell$. 22, erase: "but not divisible by 4 ". The first sentence on $\ell$. -10 should be: "Note that $\Delta(\sigma h)=\kappa(-1)^{r} \Delta(h)$. Indeed, since $\sigma_{+} \bar{\Delta}(h)=(-1)^{r} \bar{\Delta}(h)$, we have $\sigma \bar{\Delta}(h)=\zeta \bar{\Delta}(h)$ for some $\zeta \epsilon$ $E^{\times}$with $\sigma_{+} \zeta=\zeta$, thus $\zeta \in E_{+}^{\times}$. But $\kappa_{+}(x)=\kappa\left(N_{E_{+} / F} x\right)\left(x \in E_{+}^{\times}\right)$, and $N_{E_{+} / F} \zeta=\zeta \cdot \sigma \zeta \cdot \ldots \cdot \sigma^{r / 2-1} \zeta$ is $(-1)^{r}$." On $\ell .-7$, replace: "It ... that", by: "Note that $\kappa(\alpha)=\kappa(\operatorname{det} \alpha)$ depends only on $Z_{G}(h)=Z_{H}(h)$, and $\sigma$, and that". On $\ell$. -6 , erase " $k / 2$ ". On p. $276, \ell$. 15 , and p. $277, \ell .20$, replace

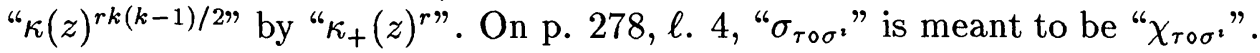
On p. $280, \ell .-22,-18,-7$, and p. $281, \ell .2,6$, replace " $\mathbb{C}^{\times k}$ " by " $\mathbb{C}^{\times r}$ ", and " $S_{k}$ " by " $S_{r}$ ". On p. $280, \ell$. -13 , replace: "Since the", by "The". On $\ell$. -11 , replace: ", it is clear", by: ". It is not necessarily true". On p. 281, erase $\ell$. 3, 4 and 5 up to: "by" (so $\ell$. 5 starts with "The"), and continue $\ell$. 7 with: "guarantees that to each spherical $\phi$ corresponds precisely one spherical $f$, and to each spherical $f$ corresponds at least one spherical $\phi . "$ This completes our correction of [F4].

The factor $\Delta_{G}^{H}(\gamma)=\Delta_{G}^{H, 1}(\gamma) \Delta_{G}^{H, 2}(\gamma)$ of [W2], p. 858, $\ell$. 1 , is now equal to our $\Delta(h)$ for $h=\gamma$, when $E / F$ is unramified, when $h_{0}$ is a $G$-regulàr element of $Z_{G}(h)=Z_{H}(h)$ such that $\bar{\Delta}\left(h_{0}\right)$ is a unit in $E^{\times}$. The factor $\Delta_{G}^{H, 1}(\gamma)$ of [W2], p. $857, \ell .-5$, is the same as $|\tilde{\Delta}(h)|$ of [F4] (and [K]), while $\Delta_{G}^{H, 2}(\gamma)$ of [W2], p. $857, \ell .-1$, is 1 if $[E: F]$ is odd (as in [K], [F4]), and is equal to the sign $\kappa_{+}\left(\bar{\Delta}(h) / \bar{\Delta}\left(h_{0}\right)\right)$ (which is 1 when $[E: F]$ is odd) if 
$[E: F]$ is even. This differs from the sign of $[\mathbf{K}]$ when $[E: F]$ is divisible by 4.

\section{Appendix. Correction to [FH].}

To increase the readability of the proof of Proposition B19, on page 709 of [FH], lines 17-19, replace the sentence which starts with "Since", by: "Put $\lambda_{\phi}(g)=\langle\check{\ell}, \phi(g)\rangle$. Since $\delta_{P}^{2}=\delta_{P^{\prime}}$, we have that $\lambda_{\phi} \in \operatorname{ind}_{P}^{G}\left(\delta_{P}\right)$. Frobenius reciprocity [BZ] implies that $\operatorname{Hom}_{G}\left(\operatorname{ind}_{P}^{G}\left(\delta_{P}\right), \mathbb{C}\right)=\operatorname{Hom}_{P}\left(\delta_{P}^{-1} \delta_{P}, \mathbb{C}\right)$. Hence the first space is one dimensional, spanned by $T(\phi)=\int_{K} \phi(k) d k$." On line 20 , "=" can be replaced by: "=T( $\left.\lambda_{\phi}\right)="$. On p. $710, \ell$. 1, replace " $\left\langle. .\right.$. " by: " $\left\langle\tilde{\rho}(p)\left(\tilde{\phi}\left(k^{\prime}\right)\right), \ell\right\rangle d_{\ell}(p) d k^{\prime} d k^{\prime}$. On line 2 , replace " $\delta_{P^{\prime}}^{-1 / 2}$ " by " $\delta_{P^{\prime}}^{1 / 2}$ ", and complete this line and the next with: " $d_{\ell}(p) d k^{\prime} d k$ ". Start $\ell$. 4 with: "(since $\left.d_{\ell}\left(p^{-1}\right)=\delta_{P^{\prime}}(p) d_{\ell}(p)\right)$ ". On $\ell$. 5 , replace " $d p$ " by " $d_{\ell}(p)$ ". On $\ell$. $7, \tilde{L}$ should be $\check{L}$. On $\ell$. 8 , insert " $\rho(K \cap H)$ " before " $\rho(p)$ ", and add " $d_{\ell}(p) d k^{\prime} d k^{\prime}$ ". Between $\ell$. 8 and 9 , insert the line: " $=\left\langle\check{\ell}, \rho\left(f^{M^{\prime}}\right) \ell\right\rangle$, where $f^{M^{\prime}}(m)=\delta_{P^{\prime}}^{1 / 2}(m) \int_{N^{\prime}} \int_{K} \int_{K} f\left(k^{\prime} m n k\right) d n d k d k^{\prime}\left(m \in M^{\prime}\right)$."

I wish to thank J. Hakim for communicating the following questions of $\mathrm{H}$. Jacquet, here answered. The $C_{c}^{\infty}$ on p. 692 stands for "compactly supported modulo the center, smooth and $K_{v}$-finite". This is used e.g. on p. 715, $\ell .1$. The last sentence in the proof of B13, p. 703, uses [H4], which is mentioned on p. 700. Propositions C7, C8, pp. 717/718, should end with "to order 2", to assure absolute convergence, and in (6), p. 722, "))" should be replaced by ") $)^{2}$ ". On p. 718 , after $\ell$. 10, we can insert: "As noted below, we have $T \backslash D=B^{\prime} \backslash G^{\prime}$, hence $E(g, \Phi, \mu, s)=\sum_{\gamma \in T \backslash D} \Phi(\gamma g, \mu, s)$, and $J(\mu, s) \Phi$ is $|\mathbf{Z}(\mathbb{A}) T \backslash \mathbf{T}(\mathbb{A})|^{-1}$ times $\int_{\mathbf{Z}(\mathbb{A}) D \backslash \mathbf{D}(\mathbb{A})} E(g, \Phi, \mu, s) d g$." Consequently, "except ... pole," on $\ell$. $12 / 13$ can be erased. Moreover, if $f$ of $\mathrm{C} 7$ matches an $f^{D}$, using the expression of p. $715, \ell .12$, it can be seen that the expression [...] of $\mathrm{C} 7$ vanishes to the order 2, that the last line of $\mathrm{C} 8$ can be erased, and that the restriction on the place $w$ in $\mathrm{C} 11$ and $\mathrm{C} 12$ can be removed. This gives a longer variant of the proofs of $\mathrm{C} 11$ and $\mathrm{C} 12$ which does not affect the result. However, in writing this paper I strove for the shortest proof, even when more - which is not necessary for the proof - could be computed.

This approach is in contrast with that of Jacquet and his team, although recently they follow arguments resembling those which I use in [FH]; see Trans. AMS 348 (1996), p. 936. In this context, the Abstract of loc. cit., p. 913 , asserts that every cuspidal $\Pi$ has a form $\phi$ with a non zero integral, but this is of course false. Also, the reference list of loc. cit., p. 938, refers to item [JR3], where the name of one of the three authors is omitted. Further, 1. -12 on p. 311 of this "[JR3]" (Duke Math. J. 70 (1993)), asserts that [FH] "is largely "based on Hakim's thesis" ". This assertion is misleading. That 
thesis computed local information ad nauseum in the context of $G L(2)$. It would take much effort to extend such an approach to $G L(n)$. The approach presented in $[\mathbf{F H}]$ is different. It shows that very little local information is needed to develop the global theory, and consequently derive the local applications. Again, this approach is different than that of Jacquet et al.

\section{References}

[B] J. Bernstein, $P$-invariant distributions on $G L(N)$ and the classification of unitary representations of $G L(N)$ (non-archimedean case), in Lie Groups Representations II, SLN 1041 (1984), 50-102.

[BDK] J. Bernstein, P. Deligne, and D. Kazhdan, Trace Paley-Wiener theorem for reductive p-adic groups, J. Analyse Math., 47 (1986), 180-192.

[BZ] J. Bernstein and A. Zelevinskii, Representations of the group $G L(n, F)$ where $F$ is a nonarchimedean local field, Russian Math. Surveys, 31 (1976), 1-68.

[Bo] A. Borel, Linear Algebraic Groups, GTM 126, Springer-Verlag, New-York (1991).

[F1] Y. Flicker, On the symmetric square. Orbital integrals, Math Annalen, 279 (1987), 173-191.

[F2] - On the symmetric square. Applications of a trace formula, Trans. Amer. Math. Soc., 330 (1992), 125-152.

[F3] , On the symmetric square. Total global comparison, J. Funct. Anal., 22 (1994), 255-278.

[F4] - On endo-lifting, Compositio Math., 67 (1988), 271-300.

[F5] - Automorphic forms on covering groups of GL(2), Invent. Math., 57 (1980), 119-182.

[F6] Report on the fundamental lemma for GL(4) and GSp(2); Automorphic forms on algebraic groups, (1996), RIMS, Kyoto.

[FK] Y. Flicker and D. Kazhdan, Metaplectic correspondence, Publ. Math. IHES., 64 (1986), 53-110.

$[\mathrm{H}] \mathrm{T}$. Hales, Unipotent representations and unipotent classes in $S L(n)$, Amer. J. Math., (1994).

[HC] Harish-Chandra, notes by G. van Dijk, Harmonic Analysis on Reductive p-adic Groups, Lecture Notes in Mathematics 162, Springer-Verlag, New-York (1970).

[K] D. Kazhdan, On lifting, in Lie groups representations II, Lecture Notes in Mathematics, Springer-Verlag, New-York, 1041 (1984), 209-249.

[KP] D. Kazhdan and S.J. Patterson, Towards a generalized Shimura correspondence, Adv. in Math., 60 (1986), 161-234.

[R] R. Rao, Orbital integrals in reductive groups, Ann. of Math., 96 (1972), 505-510.

[Ri] R. Richardson, Conjugacy classes in Lie algebras and algebraic groups, Annales of Math., 86 (1967), 1-15.

[S] J.-P. Serre, Cohomologie Galoisienne, Lecture Notes in Mathematics 5, SpringerVerlag, New-York (1965).

[Sh] J. Shalika, A theorem on semi-simple p-adic groups, Ann. of Math., 95 (1972), 226-242. 
[SS] T. Springer and R. Steinberg, Conjugacy classes, in Seminar on Algebraic Groups and Related Finite Groups, Lecture Notes in Mathematics 131, Springer-Verlag, New-York (1970).

[V] M.-F. Vigneras, Caractérisation des intégrales orbitales sur un groupe réductif padique, J. fac. sci. univ. Tokyo, 28 (1982), 945-961.

[W1] J.-L. Waldspurger, Homogeneité de certaines distributions sur les groupes p-adiques, preprint (1993).

[W2] _ Sur les intégrales orbitales tordues pour les groupes linéaires: un lemme fondamental, Canad. J. Math., 43 (1991), 852-896.

Received February 8, 1994.

The Ohio State University

Columbus, OH 43210-1174

E-mail address: flicker@math.ohio-state.edu. 
Mogens L. Hansen and Richard V. Kadison, Banach algebras with uni-

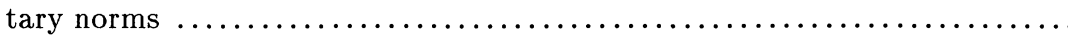

Xin-hou Hua, Sharing values and a problem due to C.C. Yang ..........

Jing-Song Huang, Harmonic analysis on compact polar homogeneous

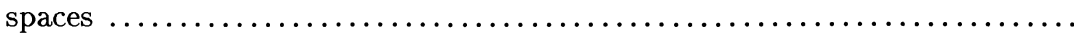

Min-Jei Huang, Commutators and invariant domains for Schrödinger prop-

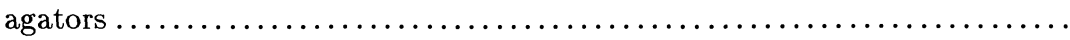

Hisao Kato, Chaos of continuum-wise expansive homeomorphisms and dy-

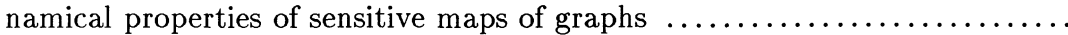

Oliver Küchle, Some properties of Fano manifolds that are zeros of sections in homogeneous vector bundles over Grassmannians ...................

Xin Li and Francisco Marcellan, On polynomials orthogonal with respect to Sobolev inner product on the unit circle $\ldots \ldots \ldots \ldots \ldots \ldots \ldots \ldots \ldots \ldots$. . . . . . .

Steven Liedahl, Maximal subfields of $Q(i)$-division rings .............. 147

Alan L.T. Paterson, Virtual diagonals and $n$-amenability for Banach algebras ....................................................

Claude Schochet, Rational Pontryagin classes, local representations, and

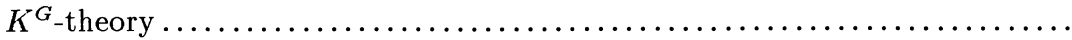

Sandra L. Shields, An equivalence relation for codimension one foliations of 3-manifolds

D. Siegel and E. O. Talvila, Uniqueness for the $n$-dimensional half space

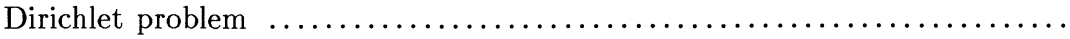

Aleksander Simonič, A Construction of Lomonosov functions and applica-

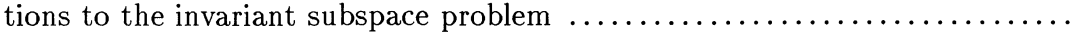

Endre Szabó, Complete intersection subvarieties of general hypersurfaces.. 


\section{PACIFIC JOURNAL OF MATHEMATICS}

Volume $175 \quad$ No. $2 \quad$ October 1996

Mean-value characterization of pluriharmonic and separately harmonic functions

LeV Abramovich AĬZEnberg, CARlos A. Berenstein and L.

WERTHEIM

Convergence for Yamabe metrics of positive scalar curvature with integral bounds on 307 curvature

\section{KAZUO AKUTAGAWA}

Generalized modular symbols and relative Lie algebra cohomology

\section{AVNER DOLNICK ASH and DAVID GINZBURG}

Convolution and limit theorems for conditionally free random variables

MaReK BożejKo, Michael LEINERT and Roland SPEICHER

$L^{p}$-bounds for hypersingular integral operators along curves

SHARAD CHANDARANA

On spectra of simple random walks on one-relator groups. With an appendix by Paul Jolissain

Pierre-Alain Cherix, Alain J. Valette and Paul Jolissaint

Every stationary polyhedral set in $\mathbf{R}^{n}$ is area minimizing under diffeomorphisms

JAIGYOUNG CHOE

Ramanujan's master theorem for symmetric cones

Hongming Ding, Kenneth I. GRoss and Donald Richards

On norms of trigonometric polynomials on SU(2)

AnTHONy H. DOOLEY and SANJiV Kumar GuPTA

On the symmetric square. Unit elements

YUVAL ZVI FLICKER

Stable constant mean curvature surfaces minimize area

KARSTEN GROSSE-BRAUCKMANN

Banach algebras with unitary norms

Mogens LEMVIG HANSEN and RiCHARD VinCENT KADISON

Harmonic analysis on compact polar homogeneous spaces

JING-SONG HUANG

Uniqueness for the $n$-dimensional half space Dirichlet problem

DAVID SIEGEL and ERIK O. TALVILA 